\title{
Is it always the same? Variability of depressive symptoms across six European countries
}

\author{
Sebastian Bernert ${ }^{\mathrm{a}, *}$, Herbert Matschinger ${ }^{\mathrm{a}}$, Jordi Alonso ${ }^{\mathrm{b}}$, Josep Maria Haro ${ }^{\mathrm{c}}$, \\ Traolach S. Brugha ${ }^{\mathrm{d}}$, Matthias C. Angermeyer ${ }^{\mathrm{e}}$ \\ the ESEMeD / MHEDEA 2000 investigators ${ }^{1}$ \\ ${ }^{a}$ University of Leipzig, Health Economics Research Unit, Department of Psychiatry, Leipzig, Germany \\ ${ }^{\mathrm{b}}$ Institut Municipal d'Investigació Mèdica (IMIM-IMAS), Health Services Research Unit, Barcelona, Spain \\ c Fundacio Sant Joan de Déu-SSM, Barcelona, Spain \\ d Section of Social and Epidemiological Psychiatry, University of Leicester, Leicester, United Kingdom \\ ${ }^{\text {e } C e n t e r ~ f o r ~ P u b l i c ~ M e n t a l ~ H e a l t h, ~ G o ̈ s i n g ~ a m ~ W a g r a m, ~ A u s t r i a ~}$
}

Received 13 August 2007; received in revised form 6 April 2008; accepted 10 April 2008

\begin{abstract}
Using common diagnostic systems together with structured interviews to assess mental disorders has made it possible to compare diagnostic groups of mental disorders across countries. The implicit assumption is that the symptomatology of a particular disorder as defined by the Diagnostic and Statistical Manual of Mental Disorders (DSM-IV) will not vary between different countries. However, it is conceivable that there will be some variability in the symptom patterns. The present study examines if differences in depressive symptom patterns across European countries can be found and if there are different associations between symptoms and the latent construct depression. Data from 4025 individuals of the European Study of the Epidemiology of Mental Disorders (ESEMeD) project were analysed. Individuals were interviewed using the Composite International Diagnostic Interview (CIDI 3.0). Confirmatory factor analysis was used to examine the associations between depressive symptoms and the latent construct of depression in each country. The proportions of endorsed symptoms of depression showed only slight variation across European countries and only minor to moderate differences in the associations between depressive symptoms and the latent construct depression. The results demonstrated that in European countries using a fully structured and standardized interview based on European-American diagnostic concepts leads to similar results with regard to depressive symptom patterns.
\end{abstract}

(C) 2008 Published by Elsevier Ireland Ltd.

Keywords: Major depression; DSM-IV; Europe; Epidemiology

\footnotetext{
* Corresponding author. Johannisallee 20, 04317 Leipzig, Germany. Tel.: +49 341 9724560; fax: +49 3419724569.

E-mail address: bernerts@gmail.com (S. Bernert).

${ }^{1}$ The ESEMeD/MHEDEA 2000 Investigators are: Jordi Alonso; Matthias C. Angermeyer; Sebastian Bernert, Ronny Bruffaerts, Traolach S. Brugha; Heather Bryson, Giovanni de Girolamo; Ron de Graaf; Koen Demyttenaere; Isabelle Gasquet; Josep Maria Haro; Steven J. Katz; Ronald C. Kessler; Viviane Kovess; Jean Pierre Lépine; Johan Ormel; Gabriella Polidori, Leo J. Russo, and Gemma Vilagut. Additional project investigators: Josué Almansa; Saena Arbabzadeh-Bouchez; Jaume Autonell; Mariola Bernal; Martine A. Buist-Bouwman; Miquel Codony; Antònia Domingo; Montserrat Ferrer; Sam S. Joo; Montserrat Martínez; Fausto Mazzi; Zoe Morgan; Pierluigi Morosini; Concepció Palacín; Josep M. Puigvert; Nick Taub; and Wilma A.M. Vollebergh.
} 


\section{Introduction}

With the development of reliable and widely accepted diagnostic systems, the Diagnostic and Statistical Manual of Mental Disorders-III (DSM-III) and the International Classification of Diseases (ICD-10), a common language in psychiatry was established. The newly developed diagnostic systems helped researchers and clinicians to overcome the previous heterogeneity of diagnostic systems used to define psychiatric cases. Structural classification systems, such as the fourth edition of the Diagnostic Statistical Manual of Mental Disorders (DSM-IV), define mental disorders by relying on the number of symptoms to establish diagnoses (Thakker and Ward, 1998). Based on these modern diagnostic systems, formal structured interviews, such as the Composite International Diagnostic Interview (CIDI) and the Structured Clinical Interview for DSM-IV (SCID), were developed to facilitate the assessment of mental disorders in a standardized way. Using common diagnostic systems together with structured interviews to assess mental disorders has made it possible to compare diagnostic groups of mental disorders across countries. This has been welcomed by epidemiologists as a major step forward in the development of the field (Dohrenwend, 1990). However, the implicit thesis of the universality of psychopathology has drawn criticism from cross-cultural psychiatrists. It has been pointed out that diagnostic categories defined in the U.S. and Western Europe may not exist, or may exist in different forms, in other cultures. Therefore, the cross-cultural application of criteria such as DSM-IV may lead to misleading results (Littlewood, 1990), a problem that has also been alluded to as "category fallacy" (Kleinman, 1977). A similar criticism has also been raised against the use of the CIDI, as this instrument was developed in the U.S. and may not be suitable to adequately capture psychopathology in nonwestern cultures (Draguns and Tanaka-Matsumi, 2003).

An implicit assumption is that the symptomatology of a particular disorder as defined by DSM-IV will not vary between different countries. However, it is conceivable that there will be some variability in symptom patterns. For example, the diagnosis of major depression according to DSM-IV requires the existence of at least five out of a total of nine depressive symptoms. Following this rule, there are a number of different symptom combinations that would lead to the same diagnosis of major depression. Thus, it is possible that in some countries psychomotor symptoms of depression are more frequent compared with other countries. This may have implications for the treatment that is required (Sobin and Sackeim, 1997), the prognosis of the depressive disorder (Loas, 1996), and the willingness of someone who suffers from this disorder to seek treatment (Galbaud du et al., 1999).

Based on data from the European Study of the Epidemiology of Mental Disorders (ESEMeD), we will address the following questions:

1. Are there differences in the frequency of reported depressive symptoms across countries using a standardized diagnostic interview based on DSM-IV?

2. Are the symptom patterns the same when applying the diagnostic criteria of DSM-IV for major depressive disorder or are there some variations between countries?

3. Is the association between symptoms and the latent construct depression the same across countries?

4. Are there variations in the variance of symptoms explained by the latent construct depression across countries?

\section{Methods}

The European Study of the Epidemiology of Mental Disorders (ESEMeD) (Alonso et al., 2002, 2004) is the first large-scale epidemiological study in Europe that has employed a comparable sampling strategy together with the use of a standardized instrument translated and adapted for use in different European countries to assess a broad range of mental disorders.

\subsection{Subjects}

The ESEMeD project is a cross-sectional study based on a stratified multi-stage random sample of 21,425 adult respondents (aged 18 years and older) living in noninstitutional settings in six European countries (Belgium $N=2419$, France $N=2894$, Germany $N=3555$, Italy $N=$ 4712, Netherlands $N=2372$, Spain $N=5473$ ). Owing to differences in access to specific population data, the sampling frame and the number of sampling stages used to obtain the final sample differed across countries. In all countries, except for France and the Netherlands, the first stage consisted of municipalities, which were stratified by region, population size or both. Selection of municipalities was proportional to size within strata and was either systematic or random depending on the country. In France, the first stage consisted of a list of telephone numbers, stratified by region and size of the cities. In the Netherlands, the first stage consisted of a random sample of addresses selected from the postal registries, with each address having the same probability of selection. In Germany and the Netherlands, the second stage units already consisted of a random selection of individuals. 
The other countries that participated in the study required one or more additional stages before the selection of the individuals for interview. Thus, three sampling stage units were required to obtain the final sample in Belgium, France and Italy. In Spain, four different stages were used. The second stage consisted of the systematic selection of census tracts from the municipalities selected in the first stage. The third stage consisted of the systematic selection of households within each census tract. The interviewer had to make a list of all households in the census tract, systematically select six households, and secure one interview in each of them. If he or she was successful, then the census tract was completed. A more detailed description of the methods and the participants in the ESEMeD project is provided elsewhere (Alonso et al., 2004). The overall response rate for the six countries was $61.2 \%$, with the highest rates in Spain (78.6\%) and Italy $(71.3 \%)$, followed by Germany $(57.8 \%)$, the Netherlands (56.4\%), and Belgium (50.6\%) and the lowest in France (45.9\%). The project is part of the World Health Organization-World Mental Health Survey Initiative (Kessler and Üstün, 2000). The analyses presented in this article are based (1) on respondents who had at least endorsed the screening questions for depressive disorders plus one out of nine symptom questions, and (2) on respondents who fulfilled the diagnostic criteria for lifetime DSM-IV major depressive disorder. The resultant sub-samples include 4025 and 2590 individuals, respectively $($ Belgium $=489 / 359$, France $=828 / 642$, Germany $=525 / 369$, Italy $=663 / 445$, Netherlands $=596 / 471$ Spain $=924 / 664)$.

\subsection{Measure}

Interviews were conducted in person using the World Mental Health Survey Initiative Version of the World Health Organization Composite International Diagnostic Interview (CIDI 3.0) (Kessler and Üstün, 2004). The CIDI 3.0 is a comprehensive, fully structured diagnostic interview for the assessment of mental disorders. It provides, by means of computerized algorithms, lifetime and current (12 months and 1 month prior to the interview) diagnoses according to the International Classification of Diseases (ICD-10) (World Health Organization, 1993) and the Diagnostic and Statistical Manual of Mental Disorders (DSM- IV) (American Psychiatric Association, 1994). It was developed to be used by trained lay interviewers. Previous versions of the CIDI have been shown to be reliable and valid (Robins et al., 1988; Wittchen, 1994; Wittchen et al., 1998). Moderate to good concordance between the CIDI 3.0 and the clinician-administered non-patient edition of the Structured Clinical Interview for DSM-IV (SCID) was found (Haro et al., 2006). The CIDI 3.0 was first produced in English and underwent a rigorous process of adaptation in order to obtain conceptually and crossculturally comparable versions in each of the target countries and languages. The translation and backtranslation of the instrument were carried out by scientists with outstanding English language skills. All translators were knowledgeable in American English and culture. An expert panel was consulted to discuss problems with regard to item wording, response categories and the adaptation to the cultural context. The expert group was formed by psychiatrists, psychologists, and other mental-health service experts. Focus groups with individuals from the general population and patients were conducted to check comprehensibility of the interview schedule. Selection of participants in the groups was governed by the premise that the individuals selected should be representative of the sampling population for the study. It was attempted to reflect the structure of the general population in the participating European countries as closely as possible. Finally, interviews were conducted that served as a test for the feasibility of the instrument in an interview situation. These interviews were carried out by staff who had not otherwise been involved in the translation process. The final forward translation was translated back into English by an independent translator, whose mother tongue is American English. The translator had no knowledge of the questionnaire. Discrepancies between the forward translation and the back translation were discussed by the expert panels. All suggested changes were then implemented in the final version of the questionnaire. All six translations of the depressive symptom questions are available as Supplementary material to the electronic publication of the manuscript.

The CIDI 3.0 is computerized in order to facilitate its administration. It includes fully structured questions on presence and persistence of clusters of psychiatric symptoms followed by probes for age of onset and lifetime course. At the beginning of the questionnaire, a screening section (a Lifetime Psychiatric Screening Instrument) was administered to all respondents. It contains screening questions for mood (i.e. Major Depression and Dysthymia) and anxiety disorders. All participants responding positively to any of the screening questions had to complete the CIDI section of the specific disorder prompted by that question. With regard to depressive disorders, the following three screening questions were asked: 'Have you ever in your life had a period lasting several days or longer when most of the day you felt sad, empty or depressed?', 'Have you ever 
had a period lasting several days or longer when most of the day you were very discouraged about how things were going in your life?' and 'Have you ever had a period lasting several days or longer when you lost interest in most things you usually enjoy like work, hobbies, and personal relationships?'. All respondents who answered positively to at least one of these questions entered the depression module of the questionnaire. The symptom questions in the depression module of the questionnaire include questions about all symptoms in nine symptom groups necessary to make a diagnosis according to the DSM-IV criteria. Probe items were asked to exclude depression symptoms that were attributable to physical causes The symptom questions ask whether there was a time in his/her life where the respondent was in a sad or depressed mood (Mood), has lost interest in most things he/she usually enjoys (Interest), has gained or lost a substantial amount of weight (Weight), has experienced sleep disturbances (Sleep), was agitated or slowed down (Activity), was tired and out of energy (Energy), has experienced feelings of guilt or worthlessness (Guilt), has lost his normal ability to concentrate (Cognitive) or has had recurrent thoughts of death or suicidal ideations (Suicide). All these symptom questions refer to the same time period of at least 2 weeks.

\subsection{Statistical analysis}

Descriptive statistics were calculated to assess the proportions of symptoms endorsed in the depression section of the interview, and confirmatory factor analysis (CFA) was used to investigate the association between symptoms and the latent construct depression. Since there was no variation in the prevalence of the mood item, it was excluded from CFA. Therefore, the CFA is based on eight depression symptoms (Interest, Weight, Sleep, Activity, Energy, Guilt, Cognition, Suicide). In order to check the dimensional structure of the dichotomous symptom variables of depression, a one-dimensional confirmatory factor model was adopted. It is assumed that these indicators measure one and only one latent dimension to be called "depression". The estimator for this type is a robust weighted least squares estimator using tetrachoric correlations. The estimation procedure is described in appendix 4 of the Technical Appendices of the Mplus software manual (Muthén, 2004). The factor model is considered a congeneric one, as loadings as well as the error variances are allowed to vary, but error correlations are restricted to be zero (Jöreskog, 1971). Testing the inequality of loadings for all six countries results in a Chi-square of $187.1, d f=26$, and a Tucker-Lewis Index of 0.880 . Since the Tucker-Lewis does not reach the threshold of 0.900 that indicates a good model fit, the assumption of a unique measurement of depression for all countries clearly has to be rejected and the model was estimated for each country separately. $R^{2}$ measures were calculated to compare the differences of variance of symptoms explained by the latent construct depression across countries. The analyses were carried out using the program Mplus (Muthén and Muthén, 2004).

\section{Results}

\subsection{Frequencies of reported symptoms}

In Table 1 the proportions of symptoms endorsed by respondents in the different countries, without the application of diagnostic algorithms, are presented. The highest rates of endorsed symptoms are found for mood symptoms (Mood). Periods of being sad or of feeling empty or depressed are reported by almost every individual in the sample. The proportions show almost no variation across countries $(95.3 \%-97.6 \%)$. Sleep disturbances (Sleep) range from a low of $85.4 \%$ in the Netherlands to a high of $90.7 \%$ in France; cognitive disturbances (Cognitive) range from a low of $76.4 \%$ in Germany to a high of $87.9 \%$ in the Netherlands. Moderate proportions of endorsed symptoms are reported for the loss of the ability to concentrate (Cognitive: range from $75.6 \%$ to $87.9 \%$ ), loss of interest (Interest: range from $75.4 \%$ to $83.2 \%$ ) and feelings of guilt and worthlessness (Guilt: range from $66.5 \%$ to $82.0 \%$ ). The lowest overall rates of endorsed symptoms as well as the largest differences between countries can be found for psychomotor symptoms (Activity) where the proportion of endorsed symptoms ranges from a low of $39.0 \%$ in Germany to a high of $55.7 \%$ in Spain. The second lowest rates of endorsed symptoms are found for suicidal ideations and thoughts (Suicide), ranging from a low of $58.4 \%$ in Italy to a high of $69.6 \%$ in France.

In Table 1, the proportions of symptoms endorsed by those respondents who fulfilled the diagnostic criteria of DSM-IV for major depressive disorder are also reported. As expected, the percentages are higher for all symptoms across all countries. However, the symptom patterns are quite similar to those observed without diagnostic restrictions. Again, for instance, psychomotor symptoms (Activity) are endorsed least frequently across all countries and show the largest differences between countries. Second lowest percentages are found for suicidal ideations and thoughts (Suicide). 
Table 1

Proportions of endorsed symptoms for the total sample and for the sub-sample with DSM-IV lifetime diagnosis of major depression (MD) (\%, S.D.).

\begin{tabular}{|c|c|c|c|c|c|c|c|}
\hline \multirow[t]{3}{*}{ Symptom } & \multirow{3}{*}{ Sample } & \multirow{2}{*}{$\frac{\text { Belgium }}{(\text { Total } N=489)}$} & \multirow{2}{*}{$\frac{\text { France }}{(\text { Total } N=828)}$} & \multirow{2}{*}{$\frac{\text { Germany }}{(\text { Total } N=525)}$} & \multirow{2}{*}{$\frac{\text { Italy }}{(\text { Total } N=663)}$} & \multirow{2}{*}{$\frac{\text { The Netherlands }}{\text { (Total } N=596)}$} & \multirow{2}{*}{$\frac{\text { Spain }}{(\text { Total } N=924)}$} \\
\hline & & & & & & & \\
\hline & & $(\mathrm{MD}=359)$ & $(\mathrm{MD}=642)$ & $(\mathrm{MD}=369)$ & $(\mathrm{MD}=445)$ & $(\mathrm{MD}=471)$ & $(\mathrm{MD}=664)$ \\
\hline \multirow[t]{2}{*}{ Mood } & Total sample & $97.3(16.0)$ & $95.3(21.2)$ & $96.6(18.2)$ & $96.8(17.5)$ & $96.1(19.3)$ & $97.6(15.3)$ \\
\hline & Major depression & $99.4(7.5)$ & $96.9(17.4)$ & $98.6(11.6)$ & $97.5(15.5)$ & $97.2(16.4)$ & $98.2(13.3)$ \\
\hline \multirow[t]{2}{*}{ Interest } & Total sample & $83.2(37.4)$ & $81.9(38.5)$ & $75.4(43.1)$ & $78.9(40.8)$ & $80.7(39.5)$ & $78.1(41.4)$ \\
\hline & Major depression & $89.7(30.4)$ & $89.3(31.0)$ & $82.4(38.1)$ & $88.9(31.3)$ & $87.5(33.1)$ & $88.0(32.5)$ \\
\hline \multirow[t]{2}{*}{ Weight } & Total sample & $72.4(44.8)$ & $69.1(46.2)$ & $70.3(45.7)$ & $68.2(46.6)$ & $66.1(47.4)$ & $71.5(45.1)$ \\
\hline & Major depression & $76.9(42.2)$ & $75.2(43.2)$ & $75.9(43.0)$ & $76.6(42.3)$ & $71.8(45.1)$ & $79.8(40.2)$ \\
\hline \multirow[t]{2}{*}{ Sleep } & Total sample & $88.5(31.9)$ & $90.7(29.1)$ & $88.6(31.8)$ & $87.2(33.5)$ & $85.4(35.3)$ & $86.7(40.0)$ \\
\hline & Major depression & $94.4(22.9)$ & $95.0(21.8)$ & $92.4(26.6)$ & $92.4(30.3)$ & $89.8(30.3)$ & $92.2(26.9)$ \\
\hline \multirow[t]{2}{*}{ Activity } & Total sample & $46.0(49.9)$ & $45.8(49.9)$ & $39.0(48.8)$ & $41.8(49.4)$ & $41.1(49.2)$ & $55.7(49.7)$ \\
\hline & Major depression & $52.1(50.0)$ & $50.8(50.0)$ & $45.8(49.9)$ & $50.33(50.1)$ & $43.7(49.6)$ & $66.1(47.4)$ \\
\hline \multirow[t]{2}{*}{ Energy } & Total sample & $77.9(41.5)$ & $75.8(42.8)$ & $76.8(42.3)$ & $74.5(43.6)$ & $85.6(35.2)$ & $76.6(42.3)$ \\
\hline & Major depression & $84.1(36.6)$ & $82.6(38.0)$ & $87.5(33.1)$ & $85.8(34.9)$ & $91.9(27.2)$ & $87.0(33.6)$ \\
\hline \multirow[t]{2}{*}{ Guilt } & Total sample & $71.8(45.1)$ & $74.9(43.4)$ & $66.5(47.3)$ & $68.3(46.6)$ & $82.0(38.4)$ & $68.7(46.4)$ \\
\hline & Major depression & $76.0(42.7)$ & 80.5 (39.6) & $74.3(43.8)$ & $78.2(41.3)$ & $85.1(35.6)$ & $76.1(42.7)$ \\
\hline \multirow[t]{2}{*}{ Cognitive } & Total sample & $76.5(42.5)$ & $80.3(39.8)$ & $76.4(42.5)$ & $75.6(43.0)$ & $87.9(32.6)$ & $77.8(41.6)$ \\
\hline & Major depression & $85.0(35.8)$ & $87.2(33.4)$ & $85.9(34.8)$ & $87.6(32.9)$ & $93.6(24.4)$ & $86.9(33.7)$ \\
\hline \multirow[t]{2}{*}{ Suicide } & Total sample & $63.6(48.2)$ & $69.6(46.0)$ & $65.3(47.6)$ & $58.4(49.3)$ & $63.3(48.3)$ & $60.0(49.0)$ \\
\hline & Major depression & $71.0(45.3)$ & $76.0(42.7)$ & $72.4(44.8)$ & $63.6(48.2)$ & $67.9(46.7)$ & $68.5(46.5)$ \\
\hline
\end{tabular}

\subsection{Factor loadings on the latent construct depression}

Table 2 presents standardized factor loadings as results of the CFA using the symptom items to generate the latent factor depression. The mood item was excluded from the confirmatory factor analysis due to the fact that it shows almost no variation and, therefore, does not discriminate between the countries. The cognitive symptom (loss of concentration) shows the highest factor loadings across countries and ranges from a low of 0.538 in Germany to a high of 0.806 in Spain and 0.803 in France. Next comes loss of energy, which ranges from 0.493 in France to 0.753 in Italy. The lowest factor loadings are found for suicidal ideations and thoughts, with a range from a low of 0.204 in the Netherlands to a high of 0.558 in Belgium (this also represents the largest difference in factor loadings across countries). Psychomotor symptoms show the second

Table 2

Factor loadings on depression (standardized loadings).

\begin{tabular}{|c|c|c|c|c|c|c|}
\hline Symptom & Belgium & France & Germany & Italy & The Netherlands & Spain \\
\hline Cognitive & 0.707 & 0.803 & 0.538 & 0.786 & 0.610 & 0.806 \\
\hline Energy & 0.665 & 0.493 & 0.674 & 0.753 & 0.668 & 0.597 \\
\hline Activity & 0.434 & 0.521 & 0.590 & 0.673 & 0.544 & 0.748 \\
\hline Interest & 0.459 & 0.539 & 0.669 & 0.678 & 0.641 & 0.669 \\
\hline Guilt & 0.499 & 0.503 & 0.549 & 0.601 & 0.439 & 0.626 \\
\hline Sleep & 0.623 & 0.383 & 0.330 & 0.478 & 0.405 & 0.419 \\
\hline Weight & 0.327 & 0.476 & 0.368 & 0.487 & 0.490 & 0.530 \\
\hline Suicide & 0.558 & 0.317 & 0.206 & 0.270 & 0.204 & 0.420 \\
\hline Chi-square test of model fit & 21.965 & 20.882 & 17.832 & 22.180 & 15.384 & 46.405 \\
\hline \multicolumn{7}{|l|}{ Value } \\
\hline$d f$ & 15 & 17 & 16 & 18 & 12 & 17 \\
\hline P-value & 0.1087 & 0.2316 & 0.3338 & 0.2237 & 0.2211 & 0.0001 \\
\hline $\mathrm{TLI}^{\mathrm{a}}$ & 0.948 & 0.981 & 0.988 & 0.993 & 0.959 & 0.953 \\
\hline RSMEA $^{\text {b }}$ & 0.031 & 0.017 & 0.015 & 0.019 & 0.022 & 0.043 \\
\hline $\mathrm{SRMR}^{\mathrm{c}}$ & 0.078 & 0.058 & 0.060 & 0.047 & 0.076 & 0.074 \\
\hline
\end{tabular}

\footnotetext{
a Tucker-Lewis Index.

b Root mean square error of approximation.

c Standardized root mean square residual.
} 
largest differences, ranging from a low of 0.434 in Belgium to a high of 0.748 in Spain. The smallest differences in factor loadings are found for feelings of guilt and worthlessness, ranging from a low of 0.439 in the Netherlands to a high of 0.626 in Spain. Looking at the simple ranking of symptom loadings across countries, it is clear that Italy and Spain, respectively, have the highest factor loadings in six out of eight symptom groups (Interest, Weight, Activity, Energy, Guilt, Cognitive), whereas Germany and Belgium have the lowest factor loadings in five symptom groups (Interest, Weight, Sleep, Activity, Cognitive) compared with the other countries. Comparing the ranking of symptom factor loadings in each country may provide some clues to the differences in symptom patterns across countries. In four out of six countries, the cognitive item shows highest factor loadings, followed by the interest item and the energy item. The factor loadings for the loss of energy item rank highest in Italy and second in Germany followed by the Netherlands and Belgium. Factor loadings for the loss of interest item rank highest in Italy and second in Germany and Spain.

\subsection{Explained variance of symptoms}

The proportion of the variance of symptoms explained by the latent construct depression is reported in Table 3.

Lowest rates of explained variance are found for the suicide item, which ranges from $4 \%$ in Germany and the Netherlands to $31 \%$ in Belgium, followed by the weight item ranging from $11 \%$ in Belgium to $28 \%$ in Spain. The highest percentage of explained variance is reported for the cognitive symptom. Explained variance ranges from a low of $29 \%$ in Germany to a high of $65 \%$ in Spain. The second highest rates of explained variance are found for energy, with a range from $24 \%$ in France to $57 \%$ in Italy. The activity item shows the largest differences of

Table 3

Explained variance by symptom $\left(R^{2}\right)$.

\begin{tabular}{lllllll}
\hline Symptom & Belgium & France & Germany & Italy & $\begin{array}{l}\text { The } \\
\text { Netherlands }\end{array}$ & Spain \\
\hline Cognitive & 0.499 & 0.644 & 0.289 & 0.617 & 0.372 & 0.650 \\
Energy & 0.442 & 0.243 & 0.454 & 0.567 & 0.447 & 0.356 \\
Interest & 0.211 & 0.290 & 0.447 & 0.460 & 0.411 & 0.448 \\
Activity & 0.188 & 0.271 & 0.348 & 0.453 & 0.295 & 0.559 \\
Guilt & 0.249 & 0.253 & 0.301 & 0.361 & 0.193 & 0.392 \\
Sleep & 0.388 & 0.146 & 0.109 & 0.228 & 0.164 & 0.175 \\
Weight & 0.107 & 0.227 & 0.136 & 0.237 & 0.240 & 0.281 \\
Suicide & 0.312 & 0.100 & 0.043 & 0.073 & 0.042 & 0.176 \\
\hline
\end{tabular}

explained variance across countries with a range from $19 \%$ in Belgium to $56 \%$ in Spain.

\section{Discussion}

\subsection{Similarities}

The data presented here show that the prevalence of depressive symptoms as assessed with the CIDI 3.0 is quite similar in the six European countries. The same applies to the symptomatology of major depressive disorder as defined by DSM-IV. Also, the association between depressive symptoms and their standardized factor loadings on the latent variable depression shows only small to moderate variations across countries. Finally, the amount of variance of the symptoms explained by the latent construct depression shows only minor differences between countries. These findings indicate that the pattern of depressive symptoms is similar rather than different across the six European countries and that the importance of single symptom groups for the diagnosis of depression does not vary a lot across these countries. Thus, measuring depressive symptoms with a standardized interview such as the CIDI 3.0 and applying a diagnostic algorithm that is defined by a structural classification system (DSM-IV) to generate diagnosis leads to quite similar results in Western European countries. This might be due to the fact that all countries under analysis are comparable to a large extent with regard to their cultural background. Our findings are in line with other studies on depression that employed the CIDI and reported relative consistency of symptom patterns across different countries (Simon et al., 2002; Weissman et al., 1996). However, this does not necessarily indicate that the presentation of depression is universal. Studies in countries with non-Western cultural background may yield quite different results.

\subsection{Differences}

Nevertheless, there are a few remarkable differences across countries that require further discussion. Although, the overall differences in factor loadings are rather small, some countries tend to be more similar to each other than others. It was found that Italy and Spain often show higher factor loadings compared with Belgium and Germany in almost all symptom groups. France and the Netherlands show moderate factor loadings in all symptom groups. Ordering the factor loadings for each symptom across countries, it was found that the factor loadings in Spain and Italy rank 
highest on the psychomotor symptom, feelings of guilt and worthlessness, weight gain or loss, loss of interest and the cognitive symptoms of depression. Compared with these high ranks in factor loadings, it was found that Belgium and Germany rank lowest on interest and weight gain or loss. In addition, Germany shows low factor loadings in suicide and sleep disturbance items. There is no general explanation for these differences in the literature about cross-cultural psychology. Nevertheless, it is worth noting that the Romance countries (Italy, Spain, France) seem to be more similar to each other as compared with the other countries (Netherlands, Germany, Belgium). Differences in the expression of mental disorders reported usually refer to differences found when comparing non-Western with Western societies or people from different ethnic groups within one country (Parker et al., 2001; Iwata and Buka, 2002). In Europe, several crossnational studies on the epidemiology of mental disorders were conducted making use of comparable assessment strategies (Lépine et al., 1997; Dowrick et al., 1999). However, none of them compared the symptomatic profile of mental disorders across countries. On the other hand, cross-cultural research has not made use of standardized diagnostic instruments to assess mental disorders. Therefore, there is still a lack of theoretical considerations regarding how to explain different symptom patterns across countries. The presented study was intended to be exploratory in nature and not explanatory, i.e. we have assessed whether there are differences in the manifestation of depressive disorders across countries without trying to explain these. More explanatory analyses should be carried out in order to investigate the conditions of these differences and their possible implications for the treatment and course of depressive disorders.

\subsection{Limitations}

The results reported here should be interpreted bearing the following limitations in mind. Although, a careful and thorough translation procedure was carried out in all of the participating countries, it is still possible that the meaning of the questions may not be the same after translating the instrument into five different languages, i.e. the semantic equivalence is assumed, but no linguistic analyses were carried out to test whether the meaning across countries is really exactly the same. Second, other contextual factors, such as stigmatisation across countries, were not assessed. Stigma might affect the willingness to report depressive symptoms depending on the different cultural contexts and, therefore, affect the sensitivity of the used instrument (Patten, 2003). Previous studies have shown that in societies where mental disorders are highly stigmatised individuals tend to express their emotional distress through somatic complaints (Kirmayer and Groleau, 2001). In some countries, low survey response rates were obtained. This could have led to a bias in reported depressive symptoms. In some surveys non-response was positively related to mental morbidity (Kessler et al., 1995) and may, therefore, have led to an underestimation of reported symptoms of depressive disorders.

\subsection{Conclusion}

The similarities found in the expression of depression in the ESEMeD project data suggest that in European countries using a fully structured and standardized interview based on European-American diagnostic concepts leads to similar results with regard to depressive symptom patterns and the importance of depressive symptoms for the diagnosis. Further investigation is needed to explain the differences in the expression of affective disorders across countries in more detail. Secondary data analysis combining the ESEMeD data with cultural variables (ecological factors, human beliefs and values) derived from other studies such as the European Value Studies (EVS, 2004) might be helpful to explain the differences across countries to a greater extent.

\section{Acknowledgements}

This project was funded by the European Commission (Contract QLG5-1999-01042); the Piemont Region (Italy), Fondo de Investigacion Sanitaria, Instituto de Salud Carlos III, Spain (FIS 00/0028), Ministerio de Ciencia Y Tecnologia, Spain (SAF 2000-158-CE), Department de Sanitat, Generalitat de Catalunya, Spain, other local agencies and by an unrestricted educational grant from GlaxoSmithKline.

The ESEMeD project is carried out in conjunction with the World Health Organization World Mental Health (WMH) Survey Initiative. We thank the WMH staff for assistance with instrumentation, fieldwork, and data analysis. These activities were supported by the US National Institute of Mental Health (R01MH070884), the John D. and Catherine T. MacArthur Foundation, the Pfizer Foundation, the US Public Health Service (R13-MH066849, R01-MH069864, and R01 DA016558), the Fogarty International Center (FIRCA R01-TW006481), the Pan American Health Organization, Eli Lilly and Company, Ortho-McNeil Pharmaceutical, Inc., GlaxoSmithKline, and Bristol-Myers Squibb. A complete list of WMH publications can be found at http://www.hcp.med.harvard.edu/wmh/. 


\section{Appendix A. Supplementary data}

Supplementary data associated with this article can be found, in the online version, at doi:10.1016/j.psychres. 2008.04.008.

\section{References}

Alonso, J., Angermeyer, M.C., Bernert, S., Bruffaerts, R., Brugha, T.S., Bryson, H., Girolamo, G., Graaf, R., Demyttenaere, K., Gasquet, I., Haro, J.M., Katz, S.J., Kessler, R.C., Kovess, V., Lépine, J.P., Ormel, J., Polidori, G., Russo, L.J., Vilagut, G., Almansa, J., ArbabzadehBouchez, S., Autonell, J., Bernal, M., Buist-Bouwman, M.A., Codony, M., Domingo-Salvany, A., Ferrer, M., Joo, S.S., MartinezAlonso, M., Matschinger, H., Mazzi, F., Morgan, Z., Morosini, P., Palacin, C., Romera, B., Taub, N., Vollebergh, W.A., 2004. Sampling and methods of the European Study of the Epidemiology of Mental Disorders (ESEMeD) project. Acta Psychiatrica Scandinavica 109 (Supplement s420), 8-20.

Alonso, J., Ferrer, M., Romera, B., Vilagut, G., Angermeyer, M., Bernert, S., Brugha, T.S., Taub, N., McColgen, Z., De Girolamo, G., Polidori, G., Mazzi, F., de Graaf, R., Vollebergh, W.A., BuistBowman, M.A., Demyttenaere, K., Gasquet, I., Haro, J.M., Palacin, C., Autonell, J., Katz, S.J., Kessler, R.C., Kovess, V., Lépine, J.P., Arbabzadeh-Bouchez, S., Ormel, J., Bruffaerts, R., 2002. The European Study of the Epidemiology of Mental Disorders (ESEMeD/MHEDEA 2000) project: rationale and methods. International Journal of Methods in Psychiatric Research 11, $55-67$.

American Psychiatric Association, 1994. Diagnostic and Statistical Manual of Mental Disorders, 4 edn. American Psychiatric Press, Washington, DC.

Dohrenwend, B.P., 1990. 'The problem of validity in field studies of psychological disorders' revisited. Psychological Medicine 20, 195-208.

Dowrick, C., Vazquez-Barquero, J.L., Wilkinson, G., Wilkinson, C., Lehtinen, V., Dalgard, O.S., Casey, P., 1999. Organizing ODIN: a case study in European academic co-operation. The ODIN Group. European Psychiatry 14, 177-181.

European Value Studies, 2004. European Value Studies. http://www. uvt.nl/evs.

Draguns, J.G., Tanaka-Matsumi, J., 2003. Assessment of psychopathology across and within cultures: issues and findings. Behaviour Research and Therapy 41, 755-776.

Galbaud du, F.G., Newman, S.C., Boothroyd, L.J., Bland, R.C., 1999. Treatment seeking for depression: role of depressive symptoms and comorbid psychiatric diagnoses. Journal of Affective Disorders 52, $31-40$.

Haro, J.M., Arbabzadeh-Bouchez, S., Brugha, T.S., de Girolamo, G., Guyer, M.E., Jin, R., Lépine, J.P., Mazzi, F., Reneses, B., Vilagut, G., Sampson, N.A., Kessler, R.C., 2006. Concordance of the Composite International Diagnostic Interview Version 3.0 (CIDI 3.0 with standardized clinical assessments in the WHO WorldMental Health surveys. International Journal of Methods in Psychiatric Research 15, 167-180.

Iwata, N., Buka, S., 2002. Race/ethnicity and depressive symptoms: a cross-cultural/ethnic comparison among university students in East Asia, North and South America. Social Science and Medicine 55, 2243-2252.

Jöreskog, K.G., 1971. Statistical analysis of sets of congeneric tests. Psychometrika 36, 109-113.
Kessler, R.C., Üstün, T.B., 2000. The World Health Organization World Mental Health 2000 Initiative. Hospital Management International 195-196.

Kessler, R.C., Üstün, T.B., 2004. The World Mental Health (WMH) Survey Initiative Version of the World Health Organization (WHO) Composite International Diagnostic Interview (CIDI). International Journal of Methods in Psychiatric Research 13, 93-121.

Kessler, R.C., Little, R.J., Groves, R.M., 1995. Advances in strategies for minimizing and adjusting for survey nonresponse. Epidemiologic Reviews 17, 192-204.

Kleinman, A., 1977. Depression, somatization, and the new crosscultural psychiatry. Social Science and Medicine 11, 3-10.

Kirmayer, L.J., Groleau, D., 2001. Affective disorders in cultural context. Psychiatric Clinics of North America 24, 465-478.

Lépine, J.P., Gastpar, M., Mendlewicz, J., Tylee, A., 1997. Depression in the community: the first pan-European study DEPRES (Depression Research in European Society). International Clinical Psychopharmacology 12, 19-29.

Littlewood, R., 1990. From categories to contexts: a decade of the new "cross-cultural psychiatry". British Journal of Psychiatry 156, 308-327.

Loas, G., 1996. Vulnerability to depression: a model centered on anhedonia. Journal of Affective Disorders 41, 39-53.

Muthén, B.O., 2004. Mplus Technical Appendices. Muthén \& Muthén, Los Angeles.

Muthén, B.O., Muthén, L.K., 2004. Mplus User's Guide. Muthén \& Muthén, Los Angeles.

Parker, G., Gladstone, G., Chee, K.T., 2001. Depression in the planet's largest ethnic group: the Chinese. American Journal of Psychiatry $158,857-864$.

Patten, S.B., 2003. International differences in major depression prevalence: what do they mean? Journal of Clinical Epidemiology $56,711-716$.

Robins, L.N., Wing, J., Wittchen, H.U., Helzer, J.E., Babor, T.F., Burke, J., Farmer, A., Jablenski, A., Pickens, R., Regier, D.A., 1988. The Composite International Diagnostic Interview. An epidemiologic instrument suitable for use in conjunction with different diagnostic systems and in different cultures. Archives of General Psychiatry 45, 1069-1077.

Simon, G.E., Goldberg, D.P., von Korff, M., Üstün, T.B., 2002. Understanding cross-national differences in depression prevalence. Psychological Medicine 32, 585-594.

Sobin, C., Sackeim, H.A., 1997. Psychomotor symptoms of depression. American Journal of Psychiatry 154, 4-17.

Thakker, J., Ward, T., 1998. Culture and classification: the cross-cultural application of the DSM-IV. Clinical Psychology Review 18, 501-529.

Weissman, M.M., Bland, R.C., Canino, G.J., Faravelli, C., Greenwald, S., Hwu, H.G., Joyce, P.R., Karam, E.G., Lee, C.K., Lellouch, J., Lépine, J.P., Newman, S.C., Rubio-Stipec, M., Wells, J.E., Wickramaratne, P.J., Wittchen, H., Yeh, E.K., 1996. Cross-national epidemiology of major depression and bipolar disorder. Journal of the American Medical Association 276, 293-299.

Wittchen, H.U., 1994. Reliability and validity studies of the WHOComposite International Diagnostic Interview (CIDI): a critical review. Journal of Psychiatric Research 28, 57-84.

Wittchen, H.U., Lachner, G., Wunderlich, U., Pfister, H., 1998. Testretest reliability of the computerized DSM-IV version of the Munich-Composite International Diagnostic Interview (M-CIDI). Social Psychiatry and Psychiatric Epidemiology 33, 568-578.

World Health Organization, 1993. Tenth revision of the international classification of diseases. Chapter V (F): Mental and Behavioral Disorders. Clinical Descriptions and Diagnostic Guidelines. World Health Organization, Geneva. 